\title{
Keeping Time with Light
}

\begin{abstract}
An all-optical clock scheme could improve time metrology standards, taking an important step toward the redefinition of the second.
\end{abstract}

\section{by Davide Calonico*}

$\mathrm{P}$ recise timekeeping is crucial to many applications, from financial transactions to GPS navigation to interferometry-based astronomy. Today's official standard of time is the Coordinated Universal Time (UTC), generated by the combined output of hundreds of cesium atomic clocks around the world [1]. Ticking at microwave frequencies, these clocks lose less than a few nanoseconds in one month. For decades, scientists have known that faster-ticking optical clocks-using light rather than microwaves - can in principle be much more accurate. Alas, these optical clocks cannot operate reliably for long times, and thus have to be coupled to "local oscillators" that keep ticking during their downtimes. These oscillators are microwave devices - typically hydrogen masers - with the intrinsic performance limitations of microwaves. Now, William Millner and colleagues at JILA and the National Institute of Standards and Technology in Colorado, in collaboration with researchers at the Physikalisch-Technische Bundesanstalt in Braunschweig, Germany, have demonstrated a scheme that replaces the microwave oscillator with an ultrastable laser [2]. This all-optical device generates a continuously running clock signal, a "timescale," that ac-

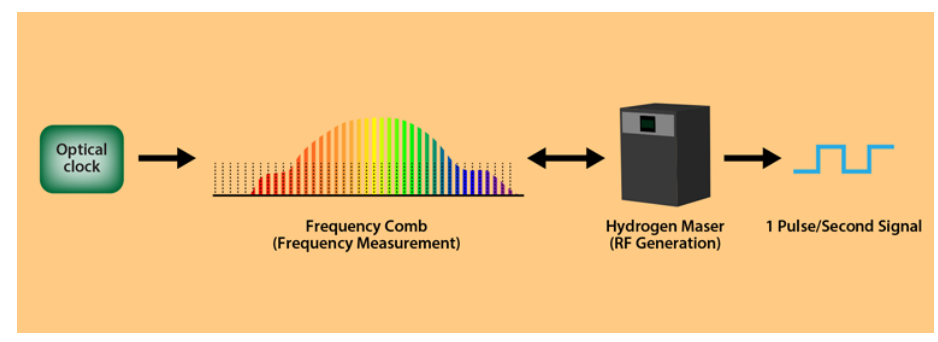

Figure 1: In a hybrid clock, an optical clock, made of a stable laser tuned to an atomic transition, produces an accurately defined frequency that is measured by a frequency comb. During the optical clock's operation, a hydrogen maser is synced with the frequency comb. During the clock's downtime, the maser acts as a freewheeling oscillator that keeps producing a timing signal. (APS/Alan Stonebraker)

*National Institute for Metrological Research (INRIM), Turin, Italy cumulates an error of order of only 100 ps over 34 days of operation-ten times better than cesium standards. With this level of reliability, the setup could provide a viable platform for improving metrological timing worldwide and for redefining the second.

The advantage of optical clocks over their microwave counterparts stems from the fact that a clock's accuracy is in principle proportional to its ticking frequency. In cesium clocks and hydrogen masers, the frequency is set to 9.2 and $1.4 \mathrm{GHz}$, respectively, by a transition between hyperfine levels of cesium and hydrogen atoms. Since the frequency of visible light is 100,000 times higher than that of microwaves, clocks based on optical transitions can be much more accurate. Optical clocks use laser light at a frequency of around $500 \mathrm{THz}$, which is adjusted to precisely match the narrow atomic resonance of particular quantum transitions of atoms such as strontium, ytterbium, and mercury, among others. A key component associated with these clocks is a so-called "femtosecond comb" - a train of pulses that can count the extremely rapid oscillations of the laser resonating with the optical transition. The comb produces a radio-frequency signal that is then used for timekeeping purposes.

Researchers have made tremendous progress with optical clocks in the last decades [3], reaching accuracies of one part in $10^{+18}$ — this value would correspond to losing less than a second over the age of the Universe. Up to now, however, the laser probing the transition didn't have sufficient longterm stability for timekeeping purposes-a feedback loop continuously steers the laser frequency so that it matches that of the optical atomic transition. This steering lacked the robustness needed to enable unsupervised operation over days or months. As a consequence, researchers developed hybrid microwave-optical systems, in which the more robust ticking of a hydrogen maser was steered to the more precise ticking of an optical clock (Fig 1). While this hybrid solution was an improvement over microwave platforms, its performance remained limited by the microwave component and fell way short of the exceptional accuracy and stability that optical clocks could warrant.This limitation still holds back the use of optical clocks for practical timekeeping applications.

The all-optical scheme demonstrated by Millner and his collaborators (Fig. 2) offers a promising way to fully tap the timekeeping potential of optical clocks. The key to their result is an all-optical, local oscillator-a laser-whose long- 


\section{Phys̄īcs}

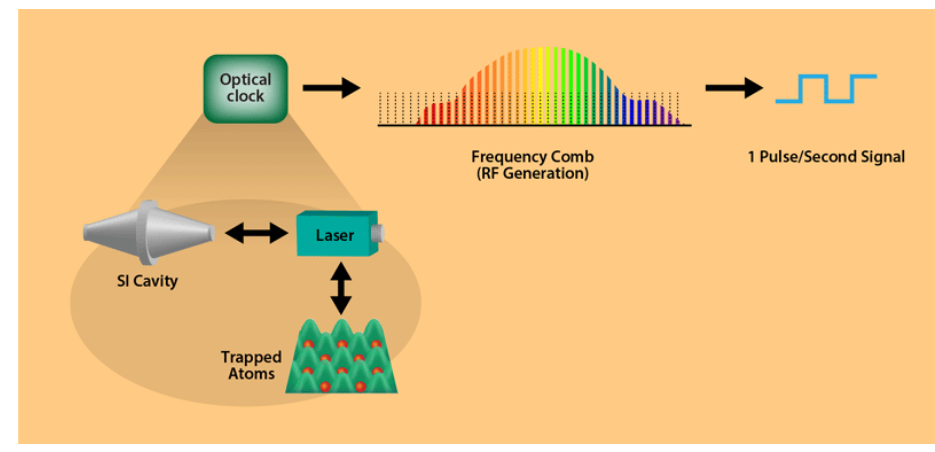

Figure 2: In the new, all-optical scheme, the core of the optical clock is an ultrastable laser, whose frequency is tuned to an optical transition of ultracold atoms and stabilized by the coupling to an external cavity. The system can run for over a month without relying on a reference microwave oscillator. (APS/Alan Stonebraker)

term stability outperforms that of a hydrogen maser. The laser is a 698-nm diode whose frequency is stabilized by coupling the laser to a 21-cm-long Fabry-Perot cavity of pure silicon kept at cryogenic temperature $(120 \mathrm{~K})$. Previous work showed that clocks based on such cavity-stabilized lasers are largely unaffected by temperature fluctuations and external perturbations like mechanical vibrations [4]. As a result, the coupling to the cavity keeps the laser-diode frequency stable at the level of one part in $10^{17}$ over the short term (up to 100 seconds) - about 1,000-10,000 better than a hydrogen maser. For many years, however, extending this performance to longer time intervals remained elusive. After 100s of seconds, the laser frequency would shift due to changes in the cavity length. While a component of this shift follows a predictable linear trend that can be corrected, other parts of the shift are nondeterministic and absolutely unpredictable.

Building on this previous work, the researchers apply several tricks-such as the use of superpolished optics, of active control of the laser power output, and of strategies for the thermal control of the environment-to further stabilize the laser frequency. The result is an ultrastable laser with unprecedented frequency predictability: the frequency evolution is accurately described by a linear drift with an exponential correction. By correcting the laser frequency data with deterministic predictions, the team evaluated the residual error of the scheme and compared it to both a strontium-87 optical clock and to a microwave atomic clock. The result is impressive: the estimated time error amounts to less than 100 ps in over a month days of operation, an order-of-magnitude improvement over the timing error of a few nanoseconds per month from the best clocks available today at metrological institutes. It is reasonable to expect that straightforward optimizations of the scheme could soon lead to a further tenfold improvement. This extrapolation indicates that extending the $10^{-18}$-level timekeeping of optical clocks from the short to the long term is now a realistic prospect.

The result is relevant for the roadmap towards the redefinition of the second in the International System of units, which envisions timescales generated by all-optical means [5]. The projected timekeeping precision of all-optical clocks would improve the spatial precision of global navigation systems, from about a meter to below a millimeter. But it could also have a profound impact on many scientific projects that rely on timing. Very Large Baseline Interferometry (VBLI), for instance, requires precise measurements of the time difference between astrophysical signals arriving at different radio telescopes. VBLI currently uses a hydrogen maser, but better timekeeping would significantly boost the resolution of VBLI observations [6]. This enhancement is particularly interesting at frequencies of hundreds of $\mathrm{GHz}$ - a spectral range recently used by the Event Horizon Telescope to capture the first image of a black hole [7]. Also, this new technology could be put to use in relativistic geodesy, which uses relativistic shifts in a clock's frequency to measure heights, monitor tides, and characterize time variations of Earth's gravity field [8]. Finally, it could enable certain dark matter searches, in which minuscule time differences between clocks at different locations, could reveal the passage of elusive dark matter particles [9].

This research is published in Physical Review Letters.

\section{REFERENCES}

[1] Time Department of the International Bureau of Weights and Measures (BIPM) https://www.bipm.org/en/bipm/tai/.

[2] Millner et al., PRL.

[3] McGrew et al., "Atomic clock performance enabling geodesy below the centimetre level," Nature 564, 87 (2018).

[4] T. Kessler et al., "A sub-40-mHz-linewidth laser based on a silicon single-crystal optical cavity," Nat. Photon. 6, 687 (2012).

[5] F. Riehle, P. Gill, F. Arias, and L. Robertsson, "The CIPM list of recommended frequency standard values: guidelines and procedures," Metrologia 55, 188 (2018).

[6] C. Clivati et al., "A VLBI experiment using a remote atomic clock via a coherent fibre link," Sci. Rep. 7, 40992 (2017).

[7] S. Doeleman (EHT Director) on behalf of the EHT Collaboration, Focus on the first Event Horizon Telescope results.

[8] J. Grotti et al., "Geodesy and metrology with a transportable optical clock," Nat. Phys. 14, 437 (2018).

[9] B. M. Roberts et al., "Search for domain wall dark matter with atomic clocks on board global positioning system satellites," Nat. Commun. 8, 1195 (2017).

10.1103/Physics.12.114 